\title{
Form preference in rats
}

GORDON M. HARRINGTON

STATE COLLEGE OF IOWA

Independently of prior prolonged experience with squares, circles or normal environment 27 hooded rats showed marked preference for circles in a shuttle box situation ( $\mathrm{p}<.0001)$. In the context of previous studies the results are interpreted as evidence of major differences in relevant variables between free choice and reinforced choice situations.

Fantz, Ordy, \& Udelf (1962) presented a convincing demonstration of a "stimulus preference" method, differential fixation, for determining sensory thresholds in infants. They emphasized the fact that the method is more sensitive "than methods providing better motivation, as by means of reward or instructions or by using a reflex response." They contended that the effectiveness of the method resulted from the use of a free choice situation eliciting an "innate non reflex response" rather than a forced or reinforced response. In this method the response is visual fixation. Summarizing a number of other studies Fantz (1961) reported innate preferences for certain visual patterns in a number of species. For example chicks without previous experience showed pecking preference for a spherical object. A natural question is whether the effectiveness of the method derives from the response used, from the availability of free choice, or both.

Infrahuman Ss in the "preference method" studies have been maintained under conditions of visual stimulus deprivation. In some cases preference changes with maturation or experience have occurred. Walk, Gibson, Pick, \& Tighe (1958) reported that rats raised in either a triangle or a circle environment alone tended on early discrimination training trials to approach the stimulus not present in the home environment. With the evidence for innate preferences in a number of species it seems reasonable to expect similar behavior in the rat. Hence these results could be interpreted as indicative of experiential establishment of differing preferences. However, since this was a discrimination learning rather than free choice situation this evidence is not conclusive.

A substantial body of work (Munn, 1950) has shown great difficulty of discrimination between square and circle for the rat. Preference method results for other discrimination situations raise the question whether the observed difficulty is a problem of visual mechanisms or a function of the test situation.

The preference of chicks for spherical objects, ethologists' observations of the functions of spot markings as releasers in a number of species, and Wada's (1961) finding of lack of habituation of cortical arousal to the eye as a stimulus all suggested the possibility that circular forms might elicit preferential behavior. This study was intended to test the possibility of an innate form preference in rats, to explore the consequences of extended experience with the forms, and to examine the characteristics of a preference method. Method

Ss were 30 INR rats pretrained to eat from the cage floor and transferred to individual cages on a single rack a few hours before their eyes opened. Animals were divided into three equal groups-"normals," "circles" and "squares." "Normals" had ordinary wire mesh front cages. "Circles" had translucent front cages, circular waterers and all cage walls and the droppings pan were decorated with black solid circles. "Squares" had identical environments except for square decorations and waterers. Ss remained in these environments until testing at 150 days of age. One "normal" and one "square" did not survive and one "square" was discarded for self injury.

Apparatus was a gray shuttle box 11 in. long $x 6$ in. deep $\times 8$ in. high with a 2-1/2 in. lateral center fence and one clear glass side. Each half of the wire grid floor was wired to a separate electronic timer through a contact relay. A 3 in. deep gray stimulus display box with vertical center partition covered the glass side. Stimulus displays were white translucent sheets, plain and one with three circles and one with three squares. Areas of circles compared with squares were identical with mirror image locations close to the center partition.

Ss were handled for $3 \mathrm{~min}$. and then habituated to the test apparatus for $400 \mathrm{sec}$. the day before the test series. For the test series Ss were placed in the box for 200 sec. for each trial and time in each half was recorded. Entry position was alternated from trial to trial. Four trials a day were given with 2-hr. intervals between trials. Stimulus conditions for the first two days were plain translucent sheets followed by two days with squares on one side and circles on the other. This four day sequence was then repeated except that circle and square positions were reversed.

\section{Results and Discussion}

The basic data were the differences in total time on a side for each animal between stimulus and no stimulus conditions. Of the $27 \mathrm{Ss}, 24$ preferred the circle side a truly substantial effect (sign test, $p<.0001$ ). Two of the three square preferences were from the normal environment group but otherwise there were no noticeable differences between groups.

The results show as substantial a sensitivity in preference effects as those reported by Fantz, Ordy, \& Udelf (1961) for human infants. A shuttle box response however seems rather more complex than fixation or chick pecking. It is possible that the rat simply fixates more on one stimulus than on the other hence adding 
to his time on that side of the box. However, since the animal is quite active in the box it seems unlikely that a simple increase in fixation time could account for such a substantial effect. It seems much more likely that there is a general preferential behavior in the presence of an innately preferred stimulus rather than a relatively simple "innate, nonreflex response." It would appear that the strength of the "preference method" rests on free choice rather than on the type of response elicited.

It is also clear that the rat could discriminate circles and squares and the magnitude of effect in these data does not betoken the visual task difficulty inferred from previous studies. The prolonged environmental experience did not elicit preferences for the alternative stimulus which have been found in the past in discrimination training. That these discrepancies arise through the use of different situations implies that the difference is more than one of sensitivity. The results suggest that free choice and reinforced choice situations may differ significantly in the behaviors they elicit. Reasonable explanations do not come readily to mind to explain why preferences or visual detections should differ between free and reinforced choice situations. A need for appropriate research is self evident.

\section{References}

Fantz, R. L., Ordy, J. M., \& Udelf, M. S. Maturation of pattern vision in infants during the first six months. $J$. comp. physiol. Psychol., 1962, 55, 907-917.

Fantz, R. L. The origin of form perception. Scient. American, 1961, $204,66-72$.

Munn, N. L. Handbook of psychological research on the rat. Boston: Houghton-Mifflin, 1950.

Wada, J. A. Modification of cortically induced responses in brain stem by shift of attention in monkeys. Science, 1961, 133, 40-42.

Walk, R. D., Gibson, E. J., Pick, H. L., \& Tighe, T. J. Further experiments on prolonged exposure to visual forms: the effect of single stimuli and prior reinforcement. J. comp. physiol. Psychol., 1958, 51, 483-487. 